\title{
Buckling of nanobeams and nanorods with cracks
}

\author{
Hina Arif ${ }^{1}$, Jaan Lellep ${ }^{2}$ \\ Department of Mathematics and Statistics, University of Tartu, Tartu, Estonia \\ E-mail: 'hina.arif@ut.ee (corresponding author)
}

\begin{abstract}
Buckling of nanobeams and nanorods is treated with the help of the nonlocal theory of elasticity. The nanobeams under consideration have piecewise constant dimensions of cross sections and are weakened with cracks or cracklike defects emanating at the re-entrant corners of steps. A general method for determination of critical buckling loads of stepped nanobeams with cracks is developed. The influence of defects on the critical buckling load is evaluated numerically and compared with similar results of other researchers.
\end{abstract}

Keywords: nanomaterial, buckling, crack.

\section{Introduction}

The nonlocal theory of elasticity has become an efficient tool for investigation of nanobeams, nanoplates and nanorods. In the present paper the nonlocal theory suggested by Eringen (2002) is applied for the determination of critical buckling loads of nanobeams and nanorods subjected to the axial compression.

The buckling of nanorods was earlier studied with the help of nonlocal theories of elasticity by Reddy (2007a, 2007b), Challamel (Challamel \& Elishakoff, 2012), Emam (2013) and others. Wang and others (Wang, Zhang, Challamel, \& Duan, 2017) developed a theory for buckling analysis of micro and nanorods making use of the Timoshenko type beam theory.

In the present paper the attention will addressed to the nanobeams of piecewise constant thicknesses. It is assumed that the nanobeams are weakened by defects which are considered as stable cracks. A method for the determination of the critical buckling loads of nanobeams based on the Euler-Bernoulli concept and the nonlocal theory of Eringen is developed.

\section{The stability problem}

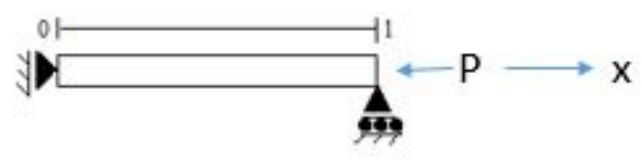

Figure 1. Simply supported nanobeam

Consider a nanobeam or nanorod of variable thickness. In the present paper it is assumed that the nanobeam of length $l$ is under the axial compression $P$ (see Figure 1). The aim of the paper is to determine the critical value of the exerted pressure which corresponds to the loss of stability of the nanobeam. For the sake of simplicity, we confine our attention to the stepped nanobeams consisting of two or three segments only. Let the nanobeam have rectangular cross section with width $b$ and thickness $h$ as

$$
h=\left\{\begin{array}{l}
h_{o}, x \in(0, a) \\
h_{1}, x \in(a, l)
\end{array} .\right.
$$

(C) 2019 Authors. Published by VGTU Press. This is an open-access article distributed under the terms of the Creative Commons Attribution (http://creativecommons.org/licenses/by/4.0/) License, which permits unrestricted use, distribution, and reproduction in any medium, provided the original author and source are credited. 
It is expected herein that the origin of the coordinate axis $O_{x}$ is located at the left-hand end of the nanobeam and $a, h_{0}, h_{1}$ are given real numbers and of course, $a<l$.

Let the nanobeam have a defect at the cross section $x=a$. The defect will be modelled as a stable crack of length $c$ which is uniformly penetrated through the width of the nanobeam.

The energy necessary for the formation of the crack will be captured in the local compliance (flexibility) of the nanobeam at the cross section $x=a$. Together with the determination of the critical buckling load we shall investigate its sensitivity with respect to the geometrical and physical parameters of the nanorods.

\section{Constitutive equations in nonlocal elasticity}

In the classical theory it is expected that the material behavior is prescribed by the Hooke's law. This means that the stress state at an arbitrary point of the body is precisely defined by the strain state at the same point (The stress tensor is proportional to the strain tensor).

In contrast to that in nonlocal theory of elasticity it is assumed that the stress state at the point of the body depends on the strain state at each point of the body. Although the first attempts to build up a continuum theory capturing seale effects were made by E. and F. Cosserat and R. Mindlin a successful approach was suggested by A. Eringen and his coworkers in the sixties of the last century. The Eringen integral constitutive equation describes the dependence between the stress at the point and the strain in the rest of domain with the help of a decaying kernel function. Eringen (2002) introduced also differential approach to the specific class of kernel functions which admitted to transform the constitutive equations into the form of differential equations. Following Eringen (2002), Reddy (2007a, 2007b), Lellep and Lenbaum (2018), we shall use the constitutive equations for the bending moment $M$ as

$$
M-\eta \frac{d^{2} M}{d x^{2}}-M_{c}=o,
$$

where $M_{c}$ denotes the bending moment in the classical theory of elasticity and

$$
\eta=\left(e_{0} a\right)^{2}
$$

in (2) $e_{0}$ is a material constant in nanomechanics, $a$ stands for the lattice spacing. It appeared that in practical cases $e_{0} a<2,1 \mathrm{~nm}$. The quantity $M_{c}$ in (2) can be evaluated as (Reddy, 2007a, 2007b)

$$
M_{c}=-E I v^{\prime \prime},
$$

where $v$ stands for the displacement in the direction of the axis $O$. Here $E I$ is the young modulus and $I$ stands for the moment of inertia of the cross section of the nanobeam. As the dimensions of the cross section are different in different sections of the nanobeam, one has

$$
I=\left\{\begin{array}{l}
\frac{b h_{0}^{3}}{12}, x \in(0, a) \\
\frac{b h_{1}^{3}}{12}, x \in(a, l)
\end{array},\right.
$$

in (5) $b$ is the width of the nanobeam.

According to the equilibrium requirements

$$
\begin{aligned}
& M^{\prime}=Q \\
& Q^{\prime}=P v^{\prime \prime},
\end{aligned}
$$

where $Q$ stands for the shear force.

It easily infers from (2), (4) that

$$
M=(\eta P-E I) \nu^{\prime \prime}
$$

The system (6), (7) can be treated as the equation

for $x \in(0, a)$ and as

$$
v^{\prime \prime}+\omega_{0}^{2} v^{\prime \prime}=0
$$

$$
v^{\prime \prime}+\omega_{1}^{2} v^{\prime \prime}=0
$$

for $x \in(a, l)$. 
In (8) and (9) the following notation is used:

$$
\omega_{0}=\sqrt{\frac{P}{E I_{0}-\eta P}},
$$

and

$$
\omega_{1}=\sqrt{\frac{P}{E I_{1}-\eta P}} .
$$

The general solutions of the fourth order differential Eqs (8) and (9) are

$$
v=A_{0} \cos \omega_{0} x+B_{0} \sin w_{0} x+C_{0} x+D_{0},
$$

and

$$
v=A_{1} \cos \omega_{1} x+B_{1} \sin w_{1} x+C_{1} x+D_{1},
$$

respectively. In (12), (13): $A_{0}, B_{0}, C_{0}, D_{0}, A_{1}, B_{1}, C_{1}, D_{1}$ stand for integration constants.

The constants will be specified according to boundary and intermediate conditions. In the case of a nanobeam simply supported at both ends the boundary conditions have the form

$$
v(0)=v(l)=0
$$

and

$$
M(0)=M(l)=0 .
$$

Taking the relations (7) into account one can present (15) as

$$
v^{\prime \prime}(0)=v^{\prime \prime}(l)=0 .
$$

\section{The local compliance}

It is evident that certain quantities (stresses, displacements) are continuous. Therefore displacement $v(x)$ must be continuous everywhere, in particular at $x=a$. Thus

$$
v(a-0)=v(a+0)
$$

In the present paper it is assumed that the generalized stresses (the bending moment $M$ and the shear force $Q$ ) are continuous. Thus,

$$
Q(a-0)=Q(a+0)
$$

and

$$
M(a-0)=M(a+0)
$$

As regards the slope of the displacement, it is accepted according to the present model that $v^{\prime}(a-0) \neq v^{\prime}(a+0)$.

Let us denote

$$
\theta=v^{\prime}(a+0)-v^{\prime}(a-0) \text {. }
$$

One can consider $\theta$ as a generalized coordinate corresponding to the generalized force $M=M(a)$. Thus one can expect that there exists the compliance function $C_{c r}$ so that

$$
\theta=C_{c r} \cdot M(a) .
$$

Several researchers among Lellep and Kraav (2016), Lellep and Liyvapuu (2016), Lellep and Lenbaum (2018) have shown that

$$
C_{c r}=\frac{72 \pi\left(1-v^{2}\right)}{E b h^{2}} f(s),
$$

and 


$$
f(s)=\int_{0}^{s} y F^{2}(y) d y,
$$

here $F=F(s)$ stands for the correction function. Corresponding values of $F(s)$ can be found from the handbook by Tada, Paris, and Irwin (2000).

\section{Critical buckling loads of simply supported nanobeams}

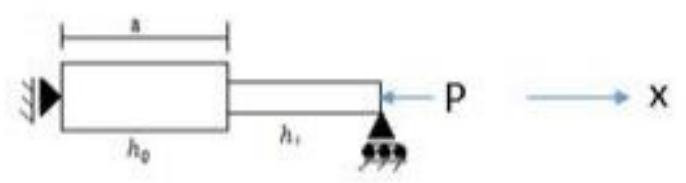

Figure 2. Stepped nanobeam

Consider the case of one-stepped nanobeam (see Figure 2) with simply supported ends in a greater detail. In the present case according to (12)

for $x \in(0, a)$ and

$$
v=A_{0} \cos \omega_{0} x+B_{0} \sin w_{0} x+C_{0} x+D_{0}
$$

$$
v=A_{1} \cos \omega_{1} x+B_{1} \sin w_{1} x+C_{1} x+D_{1}
$$

for $x \in(a, l)$.

Making use of the boundary conditions (14) and (15) with the equality (24), one obtains

$$
A_{0}=D_{0}=0 .
$$

Similarly one obtains for the segment $(a, l)$ that

$$
\begin{aligned}
& A_{1}=-B_{1} \tan \omega_{1} l, \\
& D_{1}=-C_{1} l .
\end{aligned}
$$

Substituting (26), (27) in (24), (25) admits to present the deflection of the nanobeam as

for $x \in(0, a)$ and

$$
\nu=B_{0} \sin \omega_{0} x+C_{0} x,
$$

$$
v=-\frac{B_{1}}{\cos \omega_{1} l} \sin \omega_{1}(l-x)+C_{1}(x-l)
$$

for $x \in(a, l)$.

Taking (26)-(29) into account one can present the continuity and jump conditions (18)-(22) as

$$
\begin{aligned}
& B_{0} \sin \omega_{0} a+C_{0} a+B_{2} \sin \omega_{1}(l-a)-C_{1}(a-l)=0, \\
& -B_{0} \omega_{0} \cos \omega_{0} a+C_{0}+B_{2}\left\{\omega_{1} \cos \omega_{1}(l-a)-C_{01}\left(E I_{1}-\eta P\right) \omega_{1}^{2} \sin \omega_{1}(l-a)\right\}+C_{1}=0, \\
& -B_{0} \omega_{0}^{2} \sin \omega_{0} a\left(E I_{0}-\eta P\right)-B_{2} \omega_{1}^{2} \sin \omega_{1}(l-a)\left(E I_{1}-\eta P\right)=0, \\
& -B_{0} \omega_{0}^{3} \cos \omega_{0} a\left(E I_{0}-\eta P\right)-B_{2} \omega_{1}^{3} \cos \omega_{1}(l-a)\left(E I_{1}-\eta P\right)=0,
\end{aligned}
$$

where $B_{2}=\frac{B_{1}}{\cos \omega_{1} l}$.

Calculating the determinant $\Delta$ of the system one can solve the equation $\Delta=0$ with respect to the load $P$.

\section{Numerical results}

Calculations are carried out in the case of simply supported nanorods with constant dimensions of the cross-section and in the case of one stepped nanorods. The results are accommodated in Figures 3-5. 


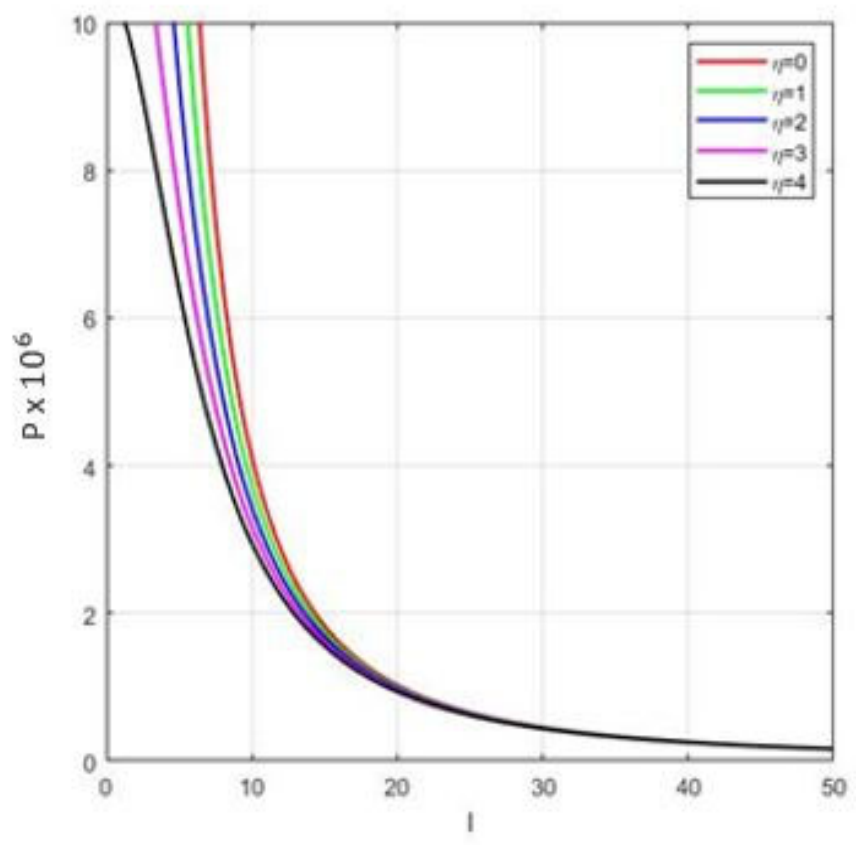

Figure 3. Critical buckling loads versus the lengths of simply supported nanobeams

In Figure 3 the relationship between the critical buckling load $P$ and the length $l$ of the nanobeam having constant thickness is shown with different values of the local parameter $\eta$. It can be seen that the values of the critical buckling load decrease monotonically with the increase in the length of the nanobeam. It also reveals that the higher is the value of the local parameter the higher will be the value of critical buckling load.

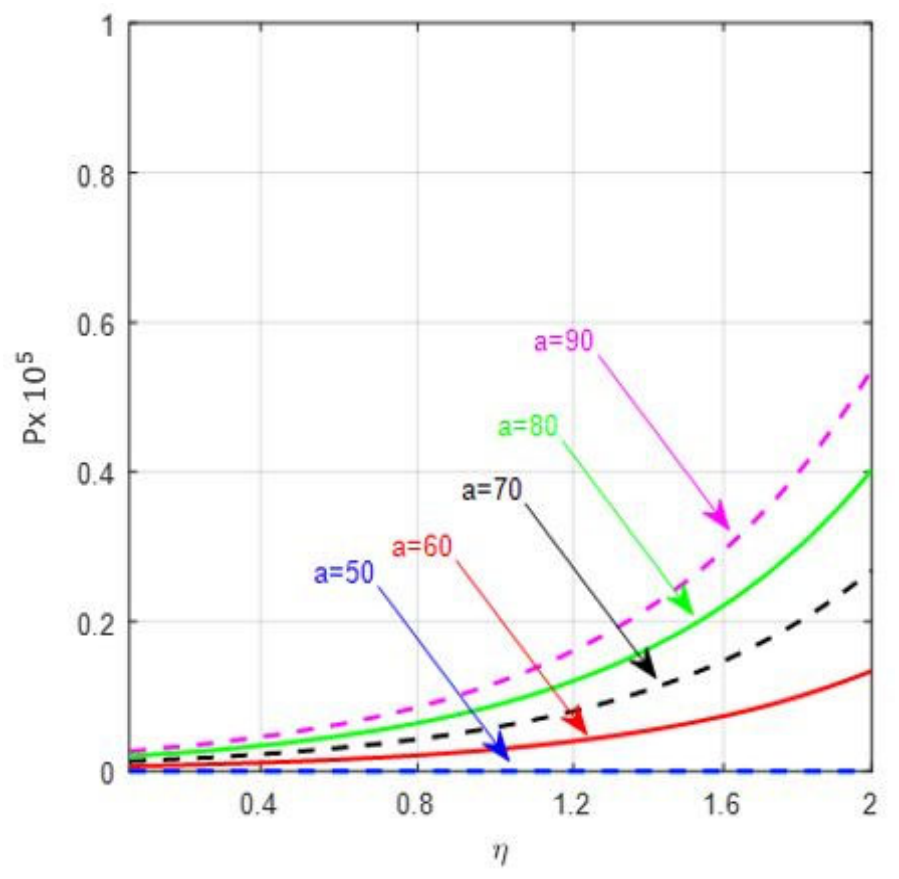

Figure 4. Critical buckling loads of stepped nanobeams versus local parameter

In Figure 4 and Figure 5 the results regarding to one-stepped simply supported nanobeams are presented.

In Figure 4 the relationship between critical buckling load $P$ and the local parameter $\eta$ is shown for different values of the crack length $a$. It can be seen from Figure 4 that there is a direct relationship between critical buckling load and the local parameter. Also, it reveals that together with the increase of the crack length the value of buckling load also increases. 


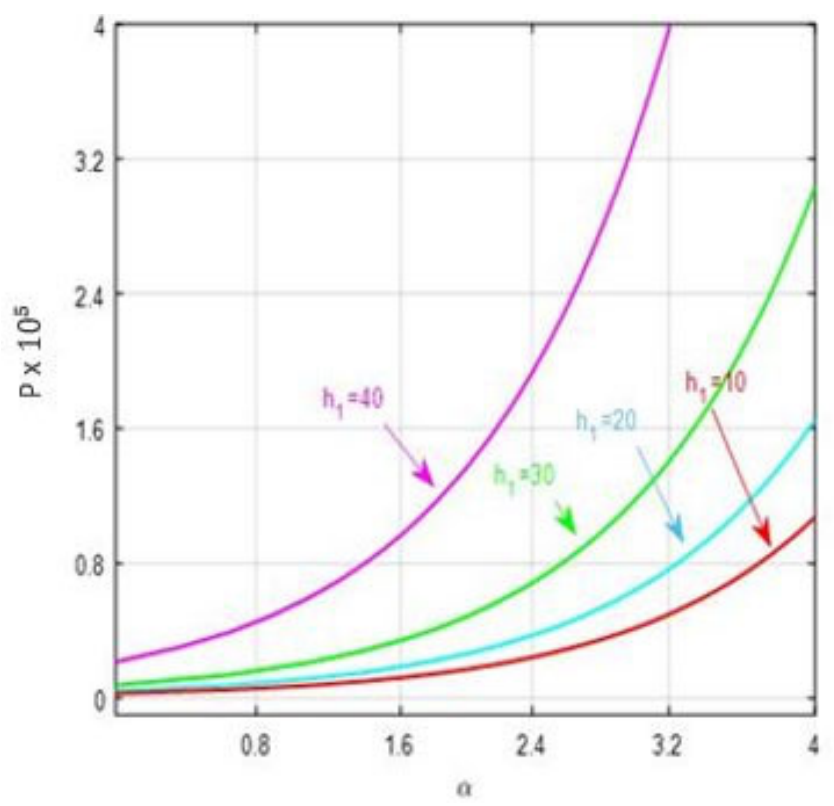

Figure 5. Critical buckling loads of stepped nanobeams with different thicknesses

In Figure 5 the relationship between critical buckling load $P$ and the step coordinat $\alpha=\frac{a}{l}$ is presented for different thicknesses of the stepped nanobeam. It is shown that the thicker is the stepped nanobeam the higher will be the value of critical buckling load; as might be expected.

\section{Conclusions}

Buckling of stepped nanobeams weakened by stable cracks was treated in the present paper. Making use of the Eringen's nonlocal theory of elasticity, method for determination of critical buckling loads was developed accounting for the influence of cracks on the stability of nanobeams. The stability of one and two stepped nanobeams subjected to the axial compression is studied. Calculations carried out revealed the results which compare favourably with the results of other researchers.

\section{Acknowledgements}

The partial support from the Institutional Research Funding IUT 20-57 and Doctoral School of Mathematics and Statistics is gratefully acknowledged.

\section{References}

Challamel, N., \& Elishakoff, J. (2012). Surface stress effects may induce softening: Euler-Bernoulli and Timoshenko buckling solutions. Physica, E: Low-dimensional Systems and Nanostructures, 44(9), 1862-1867. https://doi.org/10.1016/j.physe.2012.05.019

Emam, S. (2013). A general nonlocal nonlinear model for buckling of nanobeams. Applied Mathematical Modelling, 37(10-11), 6929-6939. https://doi.org/10.1016/j.apm.2013.01.043

Eringen, A. C. (2002). Nonlocal continuum field theories. New York: Springer.

Lellep, J., \& Kraav, T. (2016). Buckling of beams and columns with defects. International Journal of structurel Stability and Dynamics, 16(8), 1550048. https://doi.org/10.1142/S0219455415500480

Lellep, J., \& Lenbaum, A. (2018). Free vibrations of stepped nanobeams. International Journal of Computational Methods and Experimental Measurements, 6(4), 716-725. https://doi.org/10.2495/CMEM-V6-N4-716-725

Lellep, J., \& Liyvapuu, A. (2016). Natural vibrations of stepped arches. Agronomy Research, 14, 821-830.

Reddy, J. N. (2007a). Nonlocal theories for bending, buckling and vibration of beams. International Journal of Engineering Sciences, 45(2-8), 288-307. https://doi.org/10.1016/j.ijengsci.2007.04.004

Reddy, J. N. (2007b). Theory and analysis of elastic plates and shells. Boca Raton: CRC Press.

Tada, H., Paris, P. C., \& Irwin, G. R. (2000). The stress analysis of cracks handbook. New York: ASME Press. https://doi.org/10.1115/1.801535

Wang, C. M., Zhang, H., Challamel, N., \& Duan, W. H. (2017). On boundary conditions for buckling and vibration of nonlocal beams. European Journal of Mechanics - A/Solids, 61, 73-81. https://doi.org/10.1016/j.euromechsol.2016.08.014 